\title{
Study on Features, Statistics, and Security Measures of Portable Operating System
}

\author{
B. Murali Krishna ${ }^{1 *}$ and B. Santhi ${ }^{2}$ \\ ${ }^{1}$ ICT, School of Computing, SASTRA University, Tirumalaisamudram, Thanjavur, Tamil Nadu; \\ muralibhatkrishna@gmail.com \\ ${ }^{2} \mathrm{CT}$, School of Computing, SASTRA University, Tirumalaisamudram, Thanjavur, Tamil Nadu; \\ shanthi@cse.sastra.edu
}

\begin{abstract}
With the increase in memory space and the need for smarter and portable computing devices, the risk for information security also increases. Comparing this with open source and portable software the life is made even easier and other extent the threat has also evenly increased. This paper briefly explains the different kinds of portable operating system available, their performance, memory usage, and applications they support and finally risk involved in using such operating system. It also portrays the comparison between some of USB operating system and explains the field where it can be applied. The threats governing the portable operating system is brought in to discussion and the security measures required to prevent the threat is also discussed.
\end{abstract}

Keywords: POS-Portable Operating System, OS-Operating System, USB-Universal Serial Bus, DOS-Disk Operating System, BASIC-Beginner's All-purpose Symbolic Instruction Code, POSIX-Portable Operating System Interface, BSD-Berkeley Software Distribution

\section{Introduction}

The term USB operating system implies to the operating system which can be installed on a USB drive, and it can completely function from the USB drive. These operating systems will be of greater use in situations where you need to travel very often, but do not want to carry the laptop to all the places. In situations of these kinds, your USB drive can be plugged-in and you can use your own operating system. These USB operating systems or Portable operating systems are persistent, that the files once created on an USB drive are still available the next time when the USB cable is used. Such operating system is secured than carrying around files on a USB drive or the raw data. When carrying the files in a drive, there gets in situation where there is need for other computer to open those files, this

\footnotetext{
* Corresponding author:

B. Murali Krishna (muralibhatkrishna@gmail.com)
}

might lead to the greater possibility of leaving behind the files in the computer. This leads to larger security risk. Instead in case of a Portable operating system, the files can be directly opened, and there is no trace of it on the host computer. The USB operating system allows multiple users to share a single system and moreover each user can have his/her own USB drive and own USB operating system. It could be of more use in places such as school, internet café, etc. Here there is a detailed description of various portable operating systems, their performance, and memory usage.

The evolution of operating system is such that in the past 30 years there has been transition between POS and traditional operating system. The transition is such that the portable operating system is changed to traditional operating system, and now the portable operating system is back 
in use again. The term traditional operating it means fixed operating system. The first computers may it be the 'micro' computer or personal computer, all these computers were operated on a POS. Here the Portable operating system used to load into RAM where in case of the present years the USB drive is used.

One of the most breathtaking inventions in the field of storage device or personal computers is the creation of hard disk. Thought there has been advancement in invention of hard drive, the floppy disk; the CD-ROM witnessed the large amount of usage in the POS. The present decade witnessed the advent of even high performance storage device like USB flash drives, CD-RW/CD-R.

\section{Related Works}

As far as portable operating system is concerned there are several papers written on POSIX standards which is a set of standards specified by IEEE for maintaining compatibility between operating system. There are several POSIX versions like POSIX.1, POSIX.1b, POSIX.1c, POSIX 2 which defines Core Services, Real-time extensions, thread extensions and Shell and Utilities respectively. While there is much focus in the interface holding the portable operating system, here we have discussed the various type of POS their working, performance and utilities and the fields where they can be utilised.

\section{Portable Operating System}

The operating system can be divided in to three categoriesDesktop replacement, Forensics, Hacking/security. There are several operating systems in present days. The below figure shows the classification of Portable Operating System. They are classified accordingly as BSD-based, Linux based, DOS-based, GNU-based, MAC OS-based, Microsoft windows-based, Open Solaris-based. The Linux-based systems are further classified as Arch Linux-based, Debian based, Knoppix based, Gento based, Mandriva based, OpenSUSE based, Red-hat based, Slackware based. The operating system Knoppix , Damm small Linux, Slax comes under desktop replacement. The only forensic operating system is Helix, and Damm vulnerable Linux, Backtrack, ophcrack are used for Hacking and security purpose. While Tiny OS, Magnet OS, Eyes OS are used for wireless sensor networks. But indeed these OS cause a serious security issue as there is no data or residue left to show that certain users have used the system.

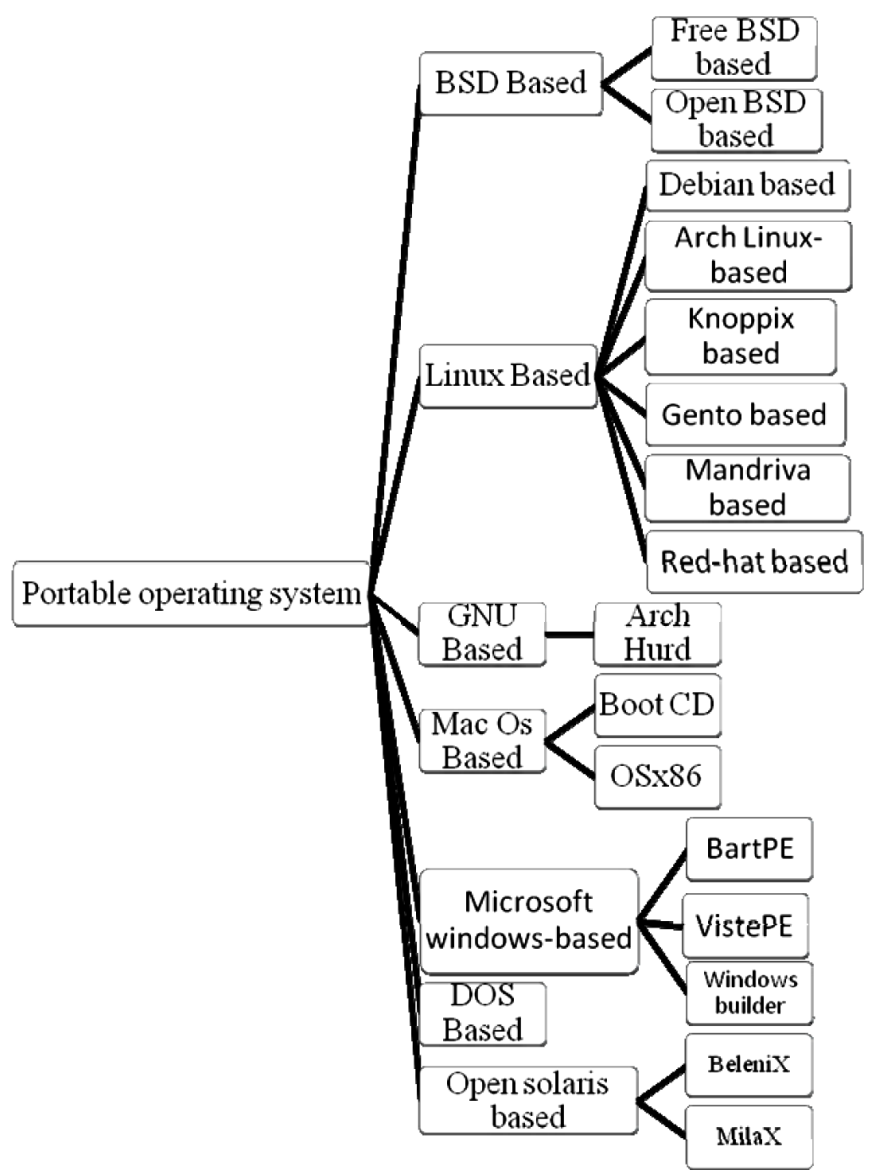

Figure 1. Classification of portable operating system.

\section{Live CD}

It was during the end of the century, the advent of 'LIVE CD' has come in to being, with which the first portable operating system was created. The operating system that runs from a CD-ROM is called a LIVE CD. It includes word processors, GUI interfaces, spreadsheets, networking and games. It is possible to create a Live CD from scratch, or already created Live CD's are available.

Today almost $100 \%$ of the computer can boot a CD. The installation process in the Linux based operating system are easier and has less impact regarding the changes it cause to the hard disk. There is no residue information left on the PC because the LIVE CD contains the entire operating system. While this causes a serious issue for information security as it provides anonymous access and violates security measures. The LIVE CD in contrast with USB operating system has the inability to store this information that is the modifications on the operating system made by the user will not be present the next time when the operating is used. The Figure-2 


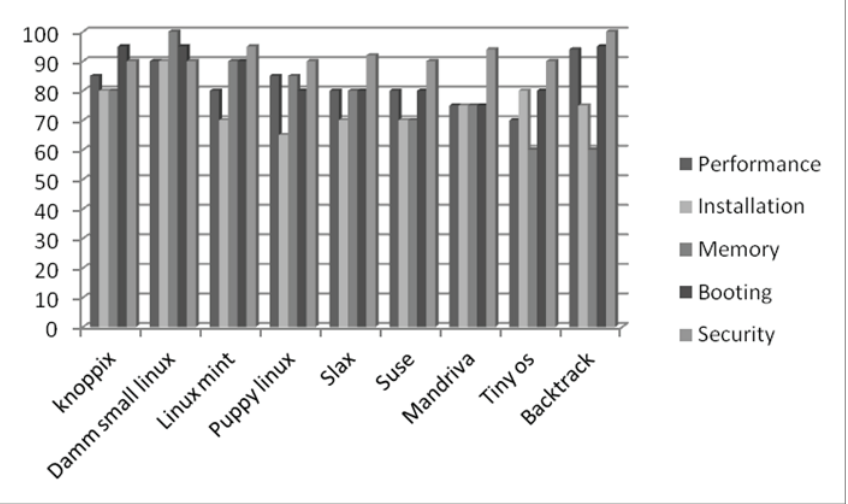

Figure 2. Comparison of different OS and its attributes.

shows the comparison based on performance, installation, memory, booting and security of portable operating system Knoppix, Damm small Linux, Linux mint, Puppy Linux, slax, suse, Mandriva, Tiny OS, Backtrack.

Here, in this figure the term performance includes testing performed to determine the stability, response on a certain workload. Installation is the availability of Live CD, the graphical user interface that is provided during installation. Memory is defined in terms of the least amount of RAM memory required to run the operating system. The booting determines how fast the operating system is available for use once it is installed or the USB is plugged in. Here everything is calculated in terms of percentage. The security defines the residual data that it leaves after usage of the application program that it allows for use.

Knoppix is one of the most efficient operating system. It is used copy files easily from hard drives with inaccessible operating systems. It consists of more than 1000 software packages included in the CD edition. It includes LXDE and KDE desktop environment, audio playback support, Internet access software, web browser (based on Mozilla Firefox), the Icedove e-mail client, GIMP, an image manipulation program, tools for data rescue and system Network analysis, administration tools, LibreOffice, a comprehensive office suite. Damm small linux is another high performance, minimum requirement distro that delivers perfect functionality and extremely fast performance on very-low-end machines, that too on a very small MB of RAM. As far as other operating system considered Damm small linux is that uses a very less amount of RAM memory. Backtrack and Damn Vulnerable Linux they both come with the same feature as Desktop replacement LIVE CD's and boot to GUI interface and they also have hundreds of security tools installed. The packages included in the Backtrack are used for network mapping, privilege escalation, covering tracks utility, maintaining access, wifi access, forensics information, Bluetooth, and reverse engineering. Though Backtrack and Damn Vulnerable Linux are one of the great POS, certain packages provided by these OS could get the users in to trouble while used on an open network. Puppy Linux uses about $165 \mathrm{MB}$ of memory and have $0-2 \%$ CPU usage. The applications available are Office: Abiword word processor, Gnumeric spreadsheets, PDF creator \& viewer, calculator, some financial tools, graphics, Internet and Multimedia applications.

The next advanced version of operating system is chromium OS. It is an open-source project that aims to build an operating system that provides a simple, fast and more secure computing experience for people who spend most of their time on the web. Chromium OS is a Linux-based operating system designed by Google to work exclusively with web applications. Google described three-tier architecture: browser, firmware, and window manager, and system-level software. It is especially built to increase the speed to use the web by increasing the booting speed. It is known for its new booting technique which is different from traditional OS. Figure 3 explains the difference between traditional OS and chromium OS in comparing how quickness of the booting speed.

Here the splash screen which is waste of time is neglected in Chromium OS compared to traditional OS. The boot loader is skipped instead jumped directly to kernel. The legacy software is simplified and browser is the ultimate point of OS. The technology used is similar to camera or mobile phones where there is no moving parts, then no mechanical head on is required to move side to side to get updated that is when Lid is closed the laptop is in sleep and when opened its ready in seconds.

\section{Strength of Portable OS}

The advantage of having USB operating system is that we can carry the entire OS and all software programs with us. The seek time and rotational latency of storage area are avoided because the USB flash drive is devoid of moving parts, so that small programs will start faster from a USB flash drive than from a local hard disk. But still the data transfer rates are slower on USB drives. With portable operating system it is easier to make changes or customise user window and applications. Install usually starts at $200 \mathrm{mb}$ 


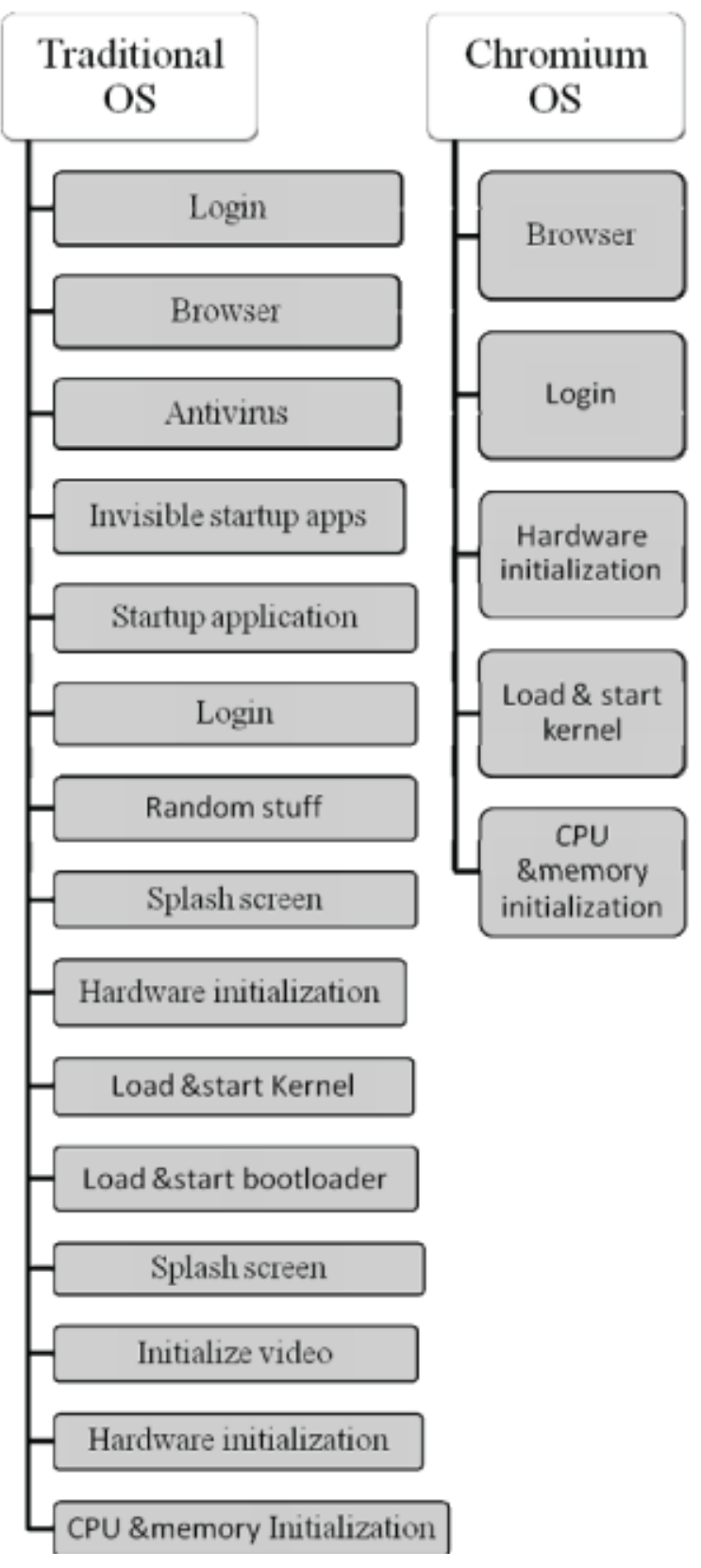

Figure 3. Difference between traditional OS and chromium OS.

and grows as the user adds applications. It provides full system encryption. The strength of using portable operating system is listed as follows.

\subsection{Reducing Malware or Virus}

While using an USB hard drive the possibility of acquiring a malware or a virus from host computer is reduced. This is because while booting an external drive the hard disk of the host compute is newer considered.

\subsection{Increased Security}

Second fact is that it provides greater security because, potentially there are very less number of people writing malware programs for this OS and its known that Linux is considered a more secure operating system than Windows.

\subsection{Secured Files}

Thirdly it increases document privacy, because you will not have to read or write files to host systems hardware since your using an USB drive.

\subsection{User Friendly Environment}

Finally it serves as a user friendly interface by allowing you to run an own customised copy of an operating system. The problem of changing configuration every time we use a different computer is reduced because all our customizations and setting will be updated on the USB.

\section{Limitations}

The POS which even though provides greater advantage they are screened by disadvantage to information security in the corporate world. If it is able to boot an USB OS then all the security measures inherent to the installed OS will be bypassed. The portable operating system includes unauthorised system tools like password breakers, and tools that scan the networks. And in last, the law enforcement or IT personnel's would be left with only evidence of having network logs that shows the IP address assigned to a certain network station that exceeded its authorized access. It will be never known or there would be no evidence whether a POS was used on the computer or not. Apparently the storage devices like USB drives, CD-ROMs on which the POS used to run, will not raise any concern among the security persons. In contrast with hardware due to the additional write cycles that occur during installation, there might be a reduction in the life of the hard drive.

\section{Security}

According to Gordon (2006) in the FBI/CSI Computer Crime and Security Survey the internal threats in an organisation is enviable to occur equally as external threat. To prevent such threats or to stop the users from using POS or USB operating system within the organisation is that, the BIOS in the computer must be set with such options that booting to CD-ROM or USB must be disabled. The BIOS 
must be password protected. In case there is a need to boot the $\mathrm{CD}$ then with authorised access the BIOS settings can be changed. This could preferably prevent the threat inside an organisation.

\section{Conclusion}

We have seen various portable operating systems, their performance, and the available software packages. Comparisons were drawn and it is known that each software serves the best for various purposes. Knnopix, Gentoo, kubuntu, xubuntu are useful for desktop purpose. Damm small Linux, Puppy Linux, Mandriva are used in places where very less memory is required for functioning, while Backtrack, Helix are used for Information security purpose. TinyOS, MagnetOs, Mantis are used for wireless sensor networks. Thought there is enormous advantage of using the Portable operating system, it also provides way for serious damage for information security. As said by Bill Gates "The first rule of any technology used in a business is that automation applied to an efficient operation will magnify the efficiency. The second is that automation applied to an inefficient operation will magnify the inefficiency." It is up to the individual to make the best use of available technology and use it for peaceful and prosperity purpose.

\section{References}

1. Polsson K (2008). A Brief Timeline of Personal Computers, Online, Available From: http://www.islandnet. com/ KPOLSSON/comphist/mini.htm

2. DUX Computer Digest: Hard Disk Drive Guide, A Brief History of the Hard Disk, Online, Available From: http:// www.duxcw.com/digest/guides/hd/hd2.htm

3. Hyslip T (2008). Thyslip Portable Operating System, Online, Available From: http://www.infosecwriters.com.

4. Sammett J E (1969). Programming languages: history and fundamentals, Prentice Hall.

5. Available From: http://en.wikipedia.org/wiki/Live_USB

6. Available From: http://wiki.remote-exploit.org/index.php/ Tools.

Table 1.

\begin{tabular}{|c|c|c|c|c|c|}
\hline Name & Creator & Cost \& availability & Windows environment & Usage & RAM usage \\
\hline Windows to go & Microsoft & Not confirmed & Windows & General, Multimedia & $120-360 \mathrm{mb}$ \\
\hline Ubuntu-Debian & Linux & Free & GNOME, KDE, Xfce, LXDE & General & $60-80 \mathrm{mb}$ \\
\hline Slax & Linux & Free & KDE Plasma Workspaces & Live & $32-320 \mathrm{mb}$ \\
\hline Knoppix & Linux & Free & LXDE & Multimedia & $60-80 \mathrm{mb}$ \\
\hline Gentoo & Linux & Free & $\begin{array}{l}\text { Enlightenment, Fluxbox, GNOME, } \\
\text { KDE, LXDE-Meta, Openbox, XBMC }\end{array}$ & Desktop & $120-320 \mathrm{mb}$ \\
\hline Kubuntu & Linux & Free & KDE Plasma Workspaces & Desktop & $120-180 \mathrm{mb}$ \\
\hline Xubuntu & Linux & Free & Xfce & Desktop & $180-256 \mathrm{mb}$ \\
\hline $\begin{array}{l}\text { Damm small } \\
\text { Linux }\end{array}$ & Linux & Free & JWM & Portable, lightweight & $50-60 \mathrm{mb}$ \\
\hline Puppy linux & Linux & Free & JWM & Lightweight.Portable & $50-60 \mathrm{mb}$ \\
\hline Suse & Linux & Free & GNOME & $\begin{array}{l}\text { Workstation and } \\
\text { server }\end{array}$ & $96-360 \mathrm{mb}$ \\
\hline Mandriva & Linux & Free & KDE Plasma Workspaces & Portable, lightweight & $128-768 \mathrm{mb}$ \\
\hline Fedora & Linux & Free & Gnome & General & $256-1024 \mathrm{mb}$ \\
\hline Backtrack & Linux & Free & $\begin{array}{l}\text { GNOME/KDE (Decided on } \\
\text { download) }\end{array}$ & Hacking/security & $256-512 \mathrm{mb}$ \\
\hline Chromium OS & Google & Free & Windows & Net book & $120-160 \mathrm{mb}$ \\
\hline Helix & Linux & Free & $\mathrm{KDE}$ & Information security & $120-160 \mathrm{mb}$ \\
\hline
\end{tabular}

